\title{
Application of the pyroelectric sensor for study of porosity effect on thermal diffusivity of nickel copper zink ferrite
}

\begin{abstract}
An investigation was carried out to study the effect of the porosity on thermal diffusivity of Nickel Copper Zinc Ferrite. The experimental technique employed to measure thermal diffusivity was the photoflash technique, and the measurements were carried out at room temperature. The heating source consists of camera flash having approximately $5 \mathrm{~ms}$ pulse duration and a thin film of polyvinylidene difluoride (PVDF) attached to the back of the samples was used as detector. Six different porosity levels were obtained by varying the grinding time following the presintering process and preceding the sintering process at $1350^{\circ} \mathrm{C}$. The porosity range obtained for the Ni0.3Cu0.1Zn0.6Fe2O4 samples was $1.8-7 \%$. The thermal diffusivity was determined from the experimental thermal transient signal. For the fitting of the experimental results, the analytical model used was based on the approximation that the pulse duration of the camera flash can be considered as Dirac-ŭ function. Thermal diffusivity values for $\mathrm{Ni0} .3 \mathrm{Cu} 0.1 \mathrm{Zn} 0.6 \mathrm{Fe} 2 \mathrm{O} 4$ samples were in the range $(7.8-8.7) \times 10-7 \mathrm{~m} 2 / \mathrm{s}$.
\end{abstract}

Keyword: Porosity; Nickel; Copper; Zinc; Ferrite; Thermal diffusivity; Cameras; $\mathrm{Ni0.3Cu0.1Zn0.6Fe2O4}$ 